\title{
Comparison on Milling Force Model Prediction of New Cold Saw Blade Milling Cutter Based on Deep Neural Network and Regression Analysis
}

\author{
Shuailiang Guo (0000-0003-4146-5616) ${ }^{1}$, Han Zheng (0000-0002-2076-9269)², Xiangzeng Liu (0000-0002-2751- \\ 6096) $)^{2}$, Lizhi Gu (0000-0002-0382-4959) ${ }^{1}$ \\ ${ }^{1}$ College of Mechanical Engineering and Automation, National Huaqiao University, Xiamen, 361021. China. Email: \\ guoshuailiang01@126.com; gulizhi888@163.com \\ ${ }^{2}$ School of Computer Science and Technology at Xidian University, Xi'an, 710071. China. Email: zhengha- \\ nhqu@163.com; xzliu@xidian.edu.cn
}

\begin{abstract}
A four factors and three levels orthogonal milling force (MF) test is designed, which qualitatively obtains the influence of four factors, namely workpiece material, workpiece diameter, milling speed and feed per tooth, on MF of the new cold saw blade milling cutter (NCSBMC), then further verifies the reliability of test data with simulation analysis of MF. The multiple linear regression analysis and deep neural network (DNN) are used to accurately fit and predict the magnitude of MF in three directions of NCSBMC, taking into account the influence of workpiece material factors on MF. Compared with the results of empirical formula, DNN has higher prediction accuracy. The research results provide theoretical guidance for the optimization of milling parameters in actual machining process.
\end{abstract}

Keywords: NCSBMC, MF, Orthogonal Test, Multiple Linear Regression Analysis, DNN

\section{Introduction}

Compared with the traditional hot saw blade milling cutter (THSBMC), NCSBMC has the advantages of high sizing accuracy, high productivity and good quality workpiece cross-section [1]. Therefore, it is widely used in the machining of various profiles, $\mathrm{pi}-$ pes and solid bar materials [2]. The teeth of NCSBMC are made of $\operatorname{Ti}(\mathrm{C}, \mathrm{N})$-based metal-ceramic material, their hardness can reach about $92 \mathrm{HRC}$, which is higher than the hardness of high-speed steel saw blade milling cutter of 60-70 HRC and carbide saw blade milling cutter of 76-82 HRC. During milling process, NCSBMC exhibits lower temperature, lower speed, larger feed and higher saw removal rate than others [3], so it has important academic significance and engineering application value for MF study of NCSBMC.

At present, MF model prediction mainly includes analytical method, empirical formula method and neural network method, each of which has its own limitations. Due to certain limitations in study of milling mechanism, individual physical parameters in milling process are difficult to accurately obtain, so it is difficult to accurately describe milling process using mathematical models, which seriously affects the prediction accuracy of analytical model [4]. The establishment of empirical formulas relies on large amounts of accurate experimental data, incidental measurement errors and experimental errors will have a great impact on establishment empirical formulas. In addition, empirical formulas rarely consider the influence of material characteristics on MF. Although the prediction accuracy of DNN model depends on training samples, DNN model itself has the characteristics of self-adaptation, self-organization, self-learning, nonlinear mapping, fault tolerance and correction capabilities, and highspeed parallel computing capabilities [5]. Since there is a high nonlinear relationship among milling parameters, workpiece material and MF, and DNN is very powerful in nonlinear modeling [6], the use of DNN model to predict MF has obvious advantage.

In traditional MF research area, S. Campocasso et al [7] performed the same heat treatment and mechanical performance treatment on specific five processing materials, and obtained the correlation coefficient between mechanical performance and cutting force through cutting experiments and mechanical performance comparison analysis. János Kundrák et al [8] studied the effect of feed on the MF and chip size ratio of face milling at different milling speeds. Geng et al [9] studied the effect of milling speed, feed per tooth and radial depth on the MF by simulating the milling of GH4169 nickel base superalloy with finite element software. $\mathrm{Li}$ et al [10] measured the force and net power of the saw blade during sawing process and obtained the distribution of sawing force within the sawing arc zone by studying the location of the point of action of the combined sawing force. Hu et al [11] reviewed the cutting force modeling method to provide a theoretical basis for cutting simulation research. Tang et al [12] calculated and studied the force and cutting power of the saw blade during cold sawing, 
and Zhu et al [13] established a transient mechanical model that can accurately describe the cut in and cut out of each tooth of the milling cutter, and concluded that the axial force can be apply to predict the wear of milling cutter. S. Turchetta and L. Sorrentino [14] used a CNC machining center to study the effect of changing machining parameters on stone cutting.

Due to the rapid development of artificial intelligence, scholars and experts have introduced neural networks to study MF. Kais I. Abdul-lateef AlAbdullah et al [15] accorded to the experimental data of artificial tissue milling with bone milling cutter, the corresponding force and temperature models were established by using artificial neural network (ANN). Li et al [16] used a neural network model to predict tool wear and cutting force, this work concluded that the neural network model is able to consider more influential factors in the machining process and has a higher prediction accuracy than the empirical formula. Wang et al [17] established a "transfer network" model for cutting force prediction based on the simulation results. Compared with the neural network model based on experimental samples, the transfer network has better performance in the case of sufficient data. However, migration learning research is currently immature and it is difficult to choose among the available training models for the particular task at hand. Ali Yeganefar et al [18] studied the prediction and optimization of surface roughness and cutting force in aluminum alloy milling operations using four methods, and this paper concluded that the neural network had higher prediction accuracy compared to support vector regression and regression analysis. The literature [15-18] showed that neural network models are very effective in predicting MF and neural network has great potential for application and development in the field of machining.

According to the literature review, we can know that there are few systematic research papers about the effect of workpiece material on MF. In this paper, workpiece material, workpiece diameter, milling speed and feed per tooth are chosen as the input variables to predict MF of NCSBMC based on regression analysis and DNN.

\section{Experiment and simulation}

\subsection{Test platform construction}

A four factors and three levels orthogonal test was designed to examine the effect of four factors on the MF of NCSBMC. The metrics are the magnitude of $F_{X}, F_{Y}$ and $F_{Z}$. The four factors and three levels for orthogonal tests are shown in Tab. 1.

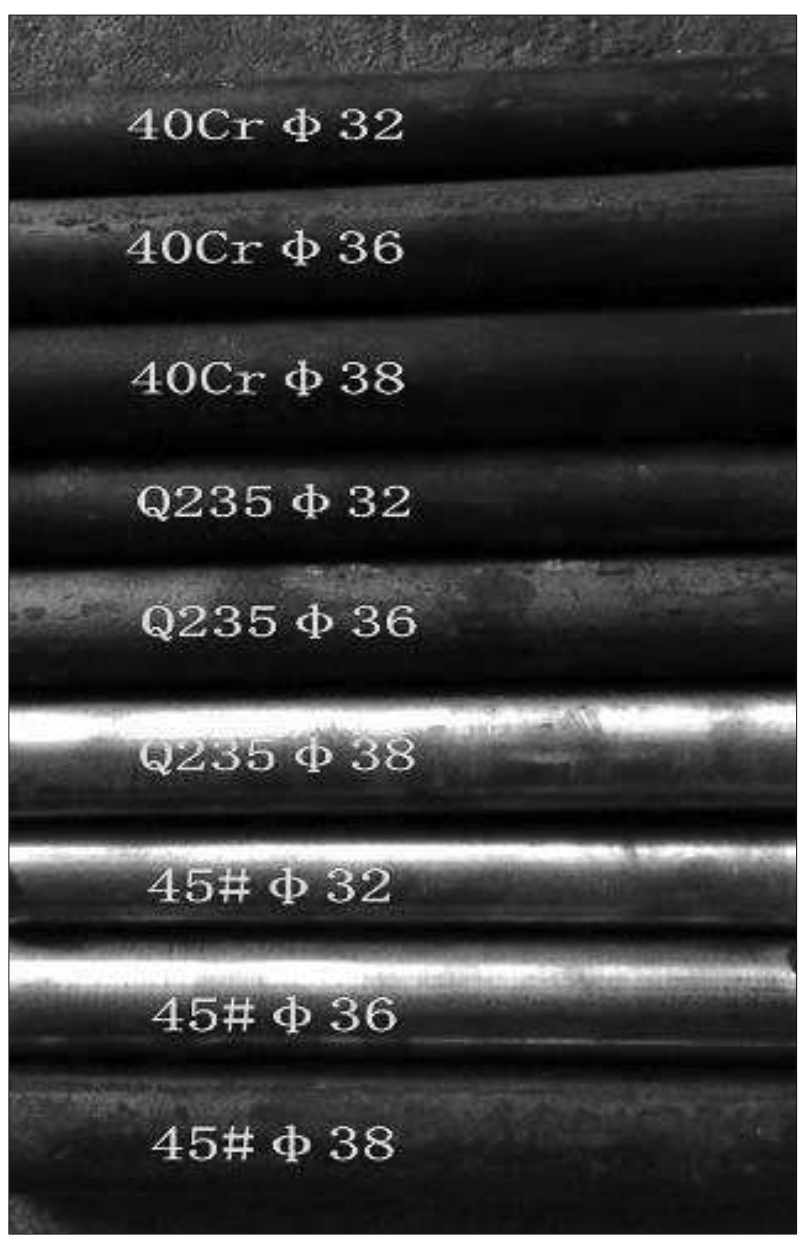

Fig. 1 Workpiece materials and diameter.

Tab. 1 Four factors and three levels orthogonal test

\begin{tabular}{ccccc}
\hline $\begin{array}{c}\text { Factors } \\
\text { Levels }\end{array}$ & $\begin{array}{c}\text { Workpiece } \\
\text { material }(\mathrm{M})\end{array}$ & $\begin{array}{c}\text { Workpiece diameter } \\
(\mathrm{D})(\mathrm{mm})\end{array}$ & $\begin{array}{c}\text { Milling speed } \\
(\mathrm{R})(\mathrm{RPM})\end{array}$ & $\begin{array}{c}\text { Feed per tooth } \\
(\mathrm{W})(\mathrm{mm})\end{array}$ \\
\hline 1 & $45 \mathrm{steel}$ & 32 & 96 & 0.020 \\
2 & $40 \mathrm{Cr}$ & 36 & 110 & 0.025 \\
3 & Q235B & 38 & 115 & 0.030 \\
\hline
\end{tabular}

A NCSBMC with 60 teeth, tool diameter $460 \mathrm{~mm}$ and saw teeth rake angle $-9^{\circ}$, rear angle $5.5^{\circ}$, was used to sawing solid round bar under dry condition. MF tests were performed on an intelligent circular sawing machine (EVERSING P-150B, power $15 \mathrm{~kW}$ ). A three-way dynamometer (Kistler 9257B) with a sampling frequency of $20 \mathrm{KHz}$ was used to obtain the milling forces in Cartesian coordinates in all three axis directions. The workpieces were placed, positioned and clamped in a self-designed fixture, which was fixed to the dynamometer by means of four M8 screws, and the dynamometer was fixed to the test platform. Workpiece materials and MF experimental device are shown in Figs. 1 and 2, respectively. 


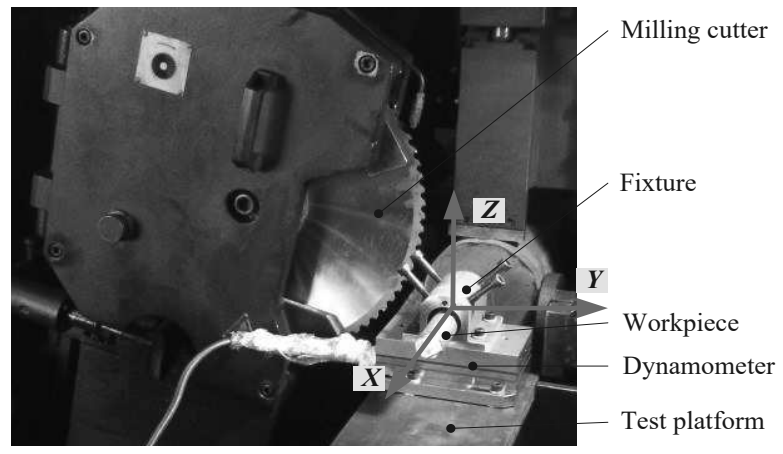

Fig. $2 \mathrm{MF}$ experimental device

\subsection{Experimental data processing and analysis}

L9 (34) was chosen to design the MF experiment, and 9 groups of machining were performed, with each group repeated three times, then the three maximum MF obtained for the $F_{X}, F_{Y}$ and $F_{Z}$ in each group were averaged to obtain MF sizes for the $F_{X}, F_{Y}$ and $F_{Z}$ of NCSBMC as shown in Tab. 2.

According to the orthogonal design data processing method, the $K$-values and the mean of the $K$-values were calculated for MF measurement data of NCSBMC, and MF numerical calculation values of NCSBMC are shown in Tab. 3.

Tab. 2 Experimental values for measuring MF of NCSBMC

\begin{tabular}{cc|c|c|c|c|c|c|c}
\hline \multirow{2}{*}{ Group } & Column & $\mathrm{M}$ & $\mathrm{D}$ & $\mathrm{R}$ & $\mathrm{W}$ & \multicolumn{3}{|c}{ Maximum MF (N) } \\
\cline { 6 - 8 } & 1 & 2 & 3 & 4 & $F_{X}$ & $F_{Y}$ & $F_{Z}$ \\
\hline 1 & $45 \#$ & 32 & 96 & 0.020 & 23.582 & 161.694 & 379.150 \\
2 & $45 \#$ & 36 & 110 & 0.025 & 24.577 & 194.348 & 484.780 \\
3 & $45 \#$ & 38 & 115 & 0.030 & 28.768 & 222.858 & 547.856 \\
4 & $40 \mathrm{Cr}$ & 32 & 110 & 0.030 & 65.111 & 233.553 & 467.518 \\
5 & $40 \mathrm{Cr}$ & 36 & 115 & 0.020 & 26.531 & 159.030 & 367.987 \\
6 & $40 \mathrm{Cr}$ & 38 & 96 & 0.025 & 33.011 & 195.684 & 474.098 \\
7 & $\mathrm{Q} 235$ & 32 & 115 & 0.025 & 37.317 & 144.701 & 419.107 \\
8 & $\mathrm{Q} 235$ & 36 & 96 & 0.030 & 40.957 & 182.088 & 526.890 \\
9 & $\mathrm{Q} 235$ & 38 & 110 & 0.020 & 43.558 & 170.894 & 424.363 \\
\hline
\end{tabular}

Tab. 3 Numerical calculation of MF for NCSBMC

\begin{tabular}{c|cccc|cccc|cccc}
\hline \multirow{2}{*}{ Factors } & \multicolumn{4}{|c|}{$F_{X}(\mathrm{~N})$} & \multicolumn{4}{c|}{$F_{Y}(\mathrm{~N})$} & \multicolumn{4}{c}{$F_{Z}(\mathrm{~N})$} \\
\cline { 2 - 13 } & $\mathrm{M}$ & $\mathrm{D}$ & $\mathrm{R}$ & $\mathrm{W}$ & $\mathrm{M}$ & $\mathrm{D}$ & $\mathrm{R}$ & $\mathrm{W}$ & $\mathrm{M}$ & $\mathrm{D}$ & $\mathrm{R}$ & $\mathrm{W}$ \\
\hline$K_{1}$ & 77 & 126 & 98 & 94 & 579 & 540 & 540 & 492 & 1412 & 1266 & 1380 & 1172 \\
$K_{2}$ & 125 & 92 & 133 & 95 & 588 & 536 & 599 & 535 & 1310 & 1380 & 1377 & 1378 \\
$K_{3}$ & 122 & 105 & 93 & 135 & 498 & 589 & 527 & 639 & 1370 & 1446 & 1335 & 1542 \\
$\overline{k_{1}}$ & 26 & 42 & 33 & 31 & 193 & 180 & 180 & 164 & 471 & 422 & 460 & 391 \\
$\overline{k_{2}}$ & 42 & 31 & 44 & 32 & 196 & 179 & 200 & 178 & 437 & 460 & 459 & 459 \\
$\overline{k_{3}}$ & 41 & 35 & 31 & 45 & 166 & 197 & 176 & 213 & 457 & 482 & 445 & 514 \\
\hline
\end{tabular}

Plotting the values of $\bar{k}_{1}, \bar{k}_{2}$ and $\bar{k}_{3}$ from Tab. 3, we can obtain the relationship between four factors and three indicators, as shown in Fig. 3. In Fig. 3, $F_{Z}$ is the largest in milling process, followed by $F_{Y}$, and $F_{X}$ is the smallest. Overall, the feed per tooth has obvious influence on $\mathrm{MF}$ in three directions. A primary and secondary analysis of the four influencing factors based on the magnitude of $F_{X}, F_{Y}$ and $F_{Z}$ fluctuations, as follows:

The influence on the $F_{X}$ is as follows: workpiece material $>$ feed per tooth $>$ milling speed $>$ working diameter.

The influence on the $F_{Y}$ is as follows: feed per tooth $>$ workpiece material $>$ milling speed $>$ working diameter.

The influence on the $F_{Z}$ is as follows: feed per tooth $>$ workpiece diameter $>$ workpiece material $>$ milling speed.

\subsection{Comparative analysis of simulation and experi-} ment

Taking the first group of parameters in Tab. 2 as an example, the experimental results and simulation results were compared and analyzed under the same conditions. The MF of NCSBMC was simulated in 3D using AdvantEdge finite element simulation software. In the simulation process, in order to reduce the grid division and calculation time, the NCSBMC model was simplified as shown in Fig. 4. After polynomial fitting to obtain MF simulation results. According to the inductive analysis of experimental data and simulation data, by taking the average value and ignoring the influence of the coordinate system setting on the positive and negative values, MF experimental data of NCSBMC is compared with the simulation data as shown in Fig. 5. 


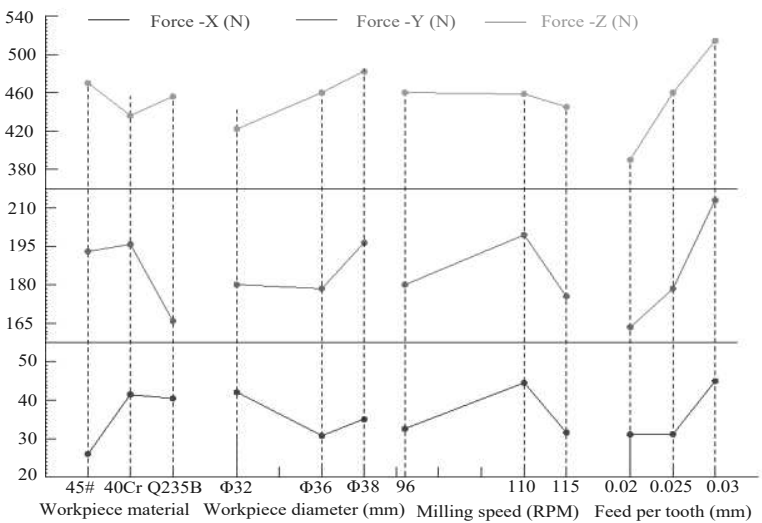

Fig. 3 Relationship between four factors and three indicators.

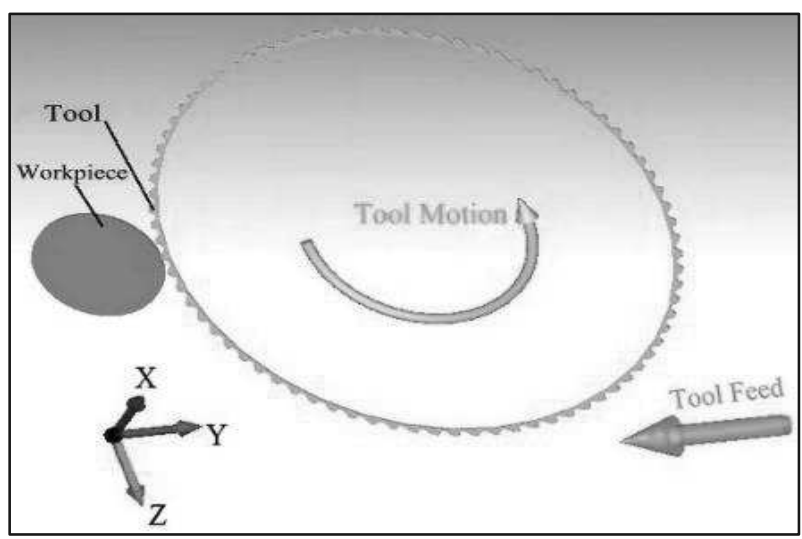

Fig. 4MF 3D simulation of NCSBMC

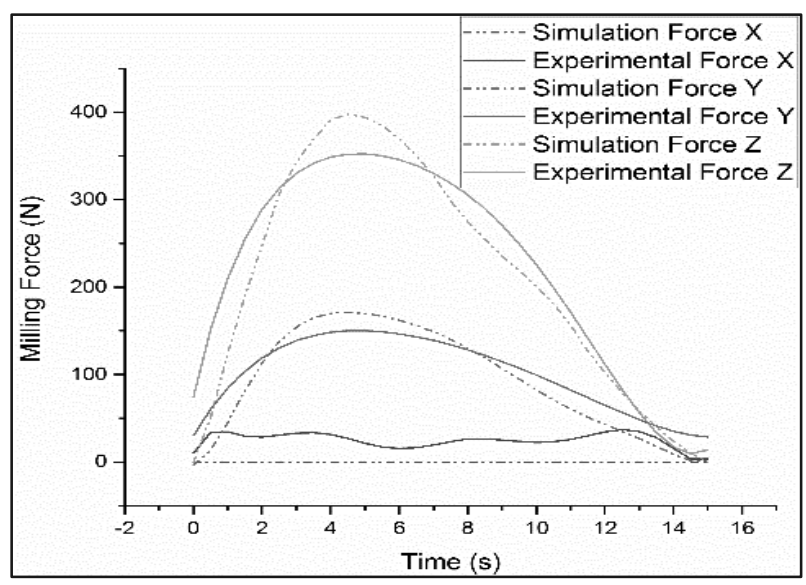

Fig. 5 Comparison of experimental and simulation values for $M F$

$$
l \mathrm{~g} F_{i}=\lg K_{i}+a_{i} \lg M+b_{i} \lg D+c_{i} \lg R+d_{i} \lg W
$$

Suppose $E=\lg F, X_{0}=\lg K, X_{1}=\lg M, X_{2}=\lg D$, $X_{3}=\lg R, X_{4}=\lg W$, we can get Eq. (3)

$$
E_{i}=X_{0}+a_{i} X_{1}+b_{i} X_{2}+c_{i} X_{3}+d_{i} X_{4}
$$

Using the orthogonal test data in Tab. 2, the test values corresponding to the $F_{X}, F_{Y}$ and $F_{Z}$ were fitted by multiple linear regression analysis with the help of Matlab mathematical calculation software. The corresponding Rockwell hardnesses are used as fitting data for the workpiece materials, and the Rockwell hardness of 45 steel, $40 \mathrm{Cr}$, Q235B is 28HRC, 32HRC, and
Fig. 5 shows that for 45 steel with a diameter of $\varphi 32 \mathrm{~mm}$, NCSBMC takes about 15 seconds to cut off the workpiece from the entry of the teeth. For $F_{Y}$ and $F_{Z}$, the experimental and simulation curves are generally downward parabolic, and the milling time corresponding to the peak of MF is roughly between the 4th and 8th seconds, when NCSBMC is involved in milling the largest number of teeth and the largest force. For $F_{X}$, the experimental values fluctuate in the range of about $20 \mathrm{~N}$ due to external conditions such as vibration, while the simulation values are almost zero.

The peak of MF obtained from simulation is slightly larger than that obtained from experiment, and the difference of $F_{Z}$ is slightly obvious, however, by comparing simulation curve of MF with experimental curve, it can be seen that the overall trend of change is close to each other. Compared with simulation data, the accuracy of experimental data is verified, which provides accurate and reliable data for the accurate prediction of MF model.

\section{MF model prediction}

\subsection{Regression analysis}

The relationship between the milling parameters and MF can be expressed in the form of an exponential function [19-21]. There are many factors that affect MF, this paper only considers four factors: workpiece material $(\mathrm{M})$, workpiece diameter (D), milling speed (R) and feed per tooth (W). For NCSBMC, the empirical formula for the MF is shown in equation (1).

$$
F_{i}=K_{i} M^{a_{i}} D^{b_{i}} R^{c_{i}} W^{d_{i}}
$$

Where:

$F_{i}$ represents $\mathrm{MF}$ in $i$-direction $[\mathrm{N}]$,

$K_{i}$ is $i$-direction determines the combined correction coefficient factor for milling conditions, friction coefficient, etc.[-],

$a_{i}, b_{i}, c_{i}$ and $d_{i}$ are the unknowns to be solved [-].

Taking both sides of MF expression in Eq. (1)

HHRC, respectively. In order to eliminate differences between data orders and to reduce errors in data processing, the data in Tab. 2 are normalized to the corresponding values distributed between [0,1]. After fitting the solution to the value of each coefficient in the expression, the empirical formula of $F_{X}, F_{Y}$ and $F_{Z}$ can be obtained as shown in equation (4).

$$
\begin{aligned}
& F_{X}=0.52991 M^{-0.6137} D^{1.4347} R^{-0.3483} W^{-0.540} \\
& F_{Y}=4550.9284 M^{0.3818} D^{-0.0476} R^{-0.1934} W^{0.9156} \\
& F_{Z}=865.7650 M^{-0.03} D^{0.7491} R^{-0.1523} W^{0.6791}
\end{aligned}
$$


To verify the accuracy of Eq. (4), on the one hand, the prediction values of MF are compared with the experimental values, as shown in Tab. 4, where $\widehat{F}_{X}, \widehat{F}_{Y}$ and $\widehat{F}_{Z}$ represent the predicted values of MF in three axes obtained from Eq. (4). $\Delta F_{X}, \Delta F_{Y}$, and $\Delta F_{Z}$ represent the deviation values between the predicted MF and the experimental MF in Tab. 2.

$$
\begin{aligned}
& \Delta F_{X}=\hat{F}_{X}-F_{X} \\
& \Delta F_{Y}=\hat{F}_{Y}-F_{Y} \\
& \Delta F_{Z}=\hat{F}_{Z}-F_{Z}
\end{aligned}
$$

From Tab. 4, the overall MF predictions are close to the experimental values, with the best prediction accuracy for the $F_{Y}$, followed by the $F_{Z}$.

On the other hand, the empirical formula is analy- zed for the rationality of the residual error. The residual analysis diagram of the $F_{X}, F_{Y}$ and $F_{Z}$ empirical formula of NCSBMC is shown in Fig. 6, where 6(a), $6(b)$, and $6(c)$ are the results of $F_{X}, F_{Y}$ and $F_{Z}$ residual analysis, respectively.

As we can see from Fig. 6, the residuals of empirical formula for $F_{X}, F_{Y}$ and $F_{Z}$ are uniformly distributed near the zero line and within the confidence interval, which means that the regression model is in accordance with the original data. The significance test values of $F_{X}, F_{Y}$ and $F_{Z}$ regression equation are 5.3840, 39.9559 and 15.1665 respectively, which are all greater than 0 , and meet the requirement of data fit. From the values, the best prediction accuracy of the regression equation for the $F_{Y}$ is obtained, followed by the $F_{Z}$. According to Tab. 4 and Fig. 6, the empirical formula for MF of NCSBMC can be used as a theoretical refe-

\begin{tabular}{|c|c|c|c|c|c|c|}
\hline \multicolumn{4}{|c|}{ Prediction values of MF(N) } & \multicolumn{3}{|c|}{ Deviation values of $\mathrm{MF}(\mathrm{N})$} \\
\hline & $\widehat{F}_{X}$ & $\widehat{\widehat{F}_{Y}}$ & $\widehat{F}_{Z}$ & $\Delta F_{X}$ & $\Delta F_{Y}$ & $\Delta F_{Z}$ \\
\hline 1 & 16.693 & 158.500 & 367.980 & -6.889 & -3.194 & -11.170 \\
\hline 2 & 16.711 & 188.320 & 458.090 & -7.866 & -6.028 & -26.690 \\
\hline 3 & 16.114 & 220.060 & 536.250 & -12.654 & -2.798 & -11.606 \\
\hline 4 & 11.783 & 235.490 & 472.780 & -53.328 & 1.937 & 5.262 \\
\hline 5 & 17.101 & 160.170 & 389.450 & -9.430 & 1.140 & 21.463 \\
\hline 6 & 17.446 & 202.930 & 458.070 & -15.565 & 7.246 & -16.028 \\
\hline 7 & 20.382 & 147.950 & 424.450 & -16.935 & 3.249 & 5.343 \\
\hline 8 & 23.291 & 180.020 & 539.330 & -17.666 & -2.068 & 12.440 \\
\hline 9 & 29.88 & 120.660 & 417.690 & -13.678 & -50.234 & -6.673 \\
\hline
\end{tabular}
rence and guide in actual production.

Tab. 4 Prediction values and deviation values of $M F$

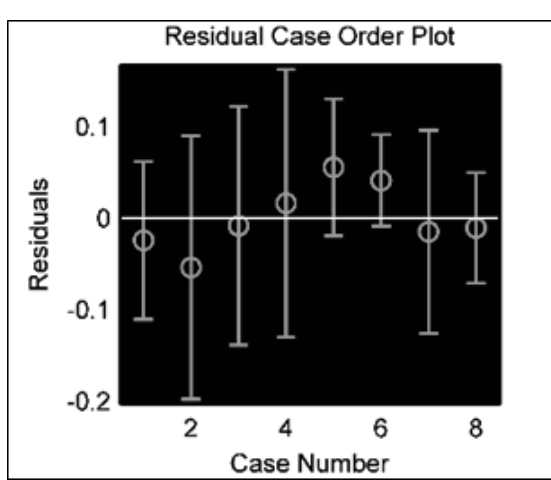

a)

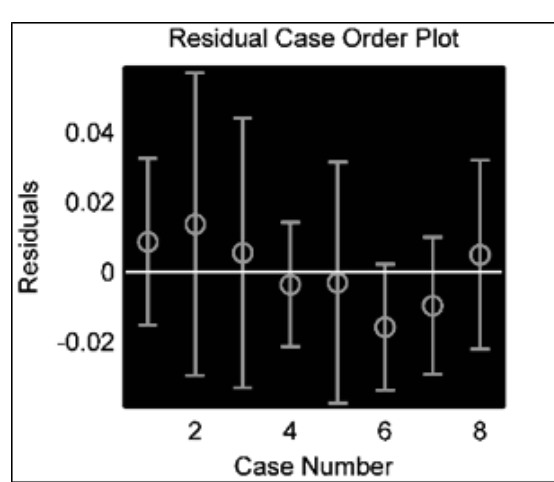

b)

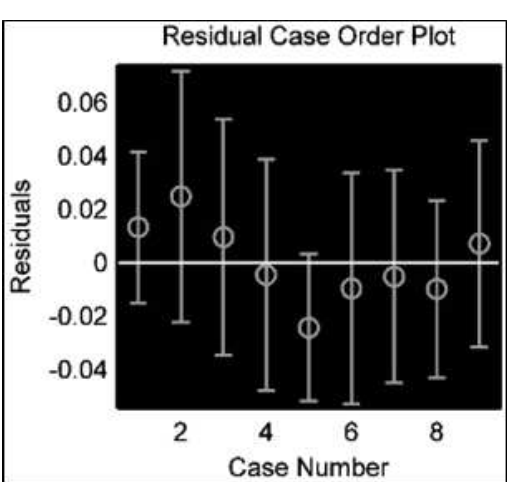

c)

Fig. 6 Residual error analysis diagram of MF empirical formula.

\subsection{DNN MF prediction}

A neural network is an extension based on a perceptual machine, while a DNN can be understood as a neural network with many hidden layers. DNN is divided by the location of different layers, neural network layers can be divided into three categories, input, hidden and output layers, and layers are fully connected. The network architecture of DNN in this paper is shown in Fig. 7, which includes an input layer, two hidden layers and an output layer. The input layer is the four characteristic values in Tab. 2. The two hidden layers both contain 64 neurons, and the output layer is the predicted value of $F_{X}, F_{Y}$ and $F_{Z}$.

Seven groups of data in Tab. 2 were selected as the sample data of the training model, and the remaining two groups were used as the test group to test the model. The MinMaxScaler normalization was performed on the input data, and the learning rate was set to 0.1. We use Relu activation as a non-linear activation function, it allows the DNN to introduce sparsity on its own, while greatly improving training speed. 


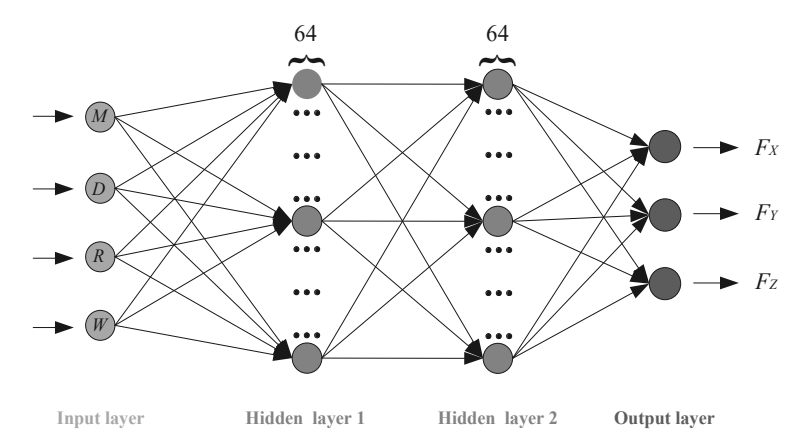

Fig. 7 Schematic of a DNN structure used in this study

DNN's forward propagation algorithm uses multiple weighted factor matrices, bias vector to perform a series of linear and activation operations on the input value vector, starting at the input layer and scaling backward layer by layer until it reaches the output layer.

The back propagation (BP) of the DNN is used to calculate the error at each layer of the forward propagation, and the weights and deviations are updated by minimizing the Loss function based on the error value.

$$
\text { Loss }=\frac{1}{2} \sum_{j \in N}\left(d_{j}-y_{j}\right)^{2}
$$

Where:

Loss using mean squared error (MSE) and Mean Absolute Error (MAE) as evaluation indicators [-],

$N$ represents all neurons in the output layer [-],

$d_{j}$ and $y_{j}$ are the expected and output values of the $j$-th neuron in the output layer, respectively [-].

In order to prevent over-fitting and excessive learning rate leading to non-convergence, and to speed up the learning process and improve the tuning efficiency, when the degree of Loss on the training set is less than a certain threshold, two methods, Early stopping and callback function (ReduceLROnPlateau), are used to stop further training and prevent the accuracy on the test set from decreasing as a result of further training. The change in the number of training sessions and the Loss value is shown in Fig. 8.

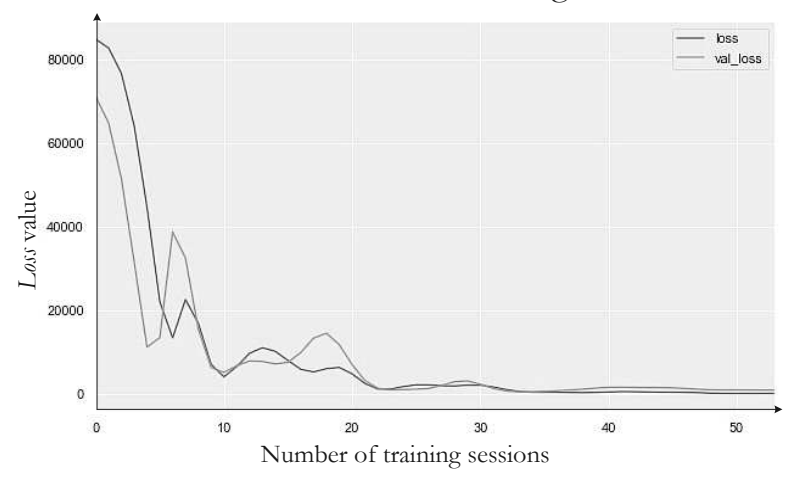

Fig. 8 Changes in the number of training sessions and Loss value

In Fig. 8, the horizontal coordinate represents the number of training sessions, the vertical coordinate represents the Loss value. The blue line represents the change in the Loss value with the number of training sessions on the training set, and the orange line represents the change in the Loss value with the number of training sessions on the validation set. As shown in Fig. 8, the Loss value converges as the number of training sessions increases.

In DNN model, the data is divided into a training set and a testset. In the case of insufficient data, in order to make full use of the data to test the effect of the algorithm, the data is divided into five groups, one of which is used as a testset at a time, while the remaining four groups are used as a training set. The $K$-fold cross-validation each data has only one chance to be classified into the training or testset during each iteration, the validation and testset alternate with each other to form a complementary set loop, with the result of the model equal to the average of four iterations of MSE and MAE, as shown in Fig. 9.

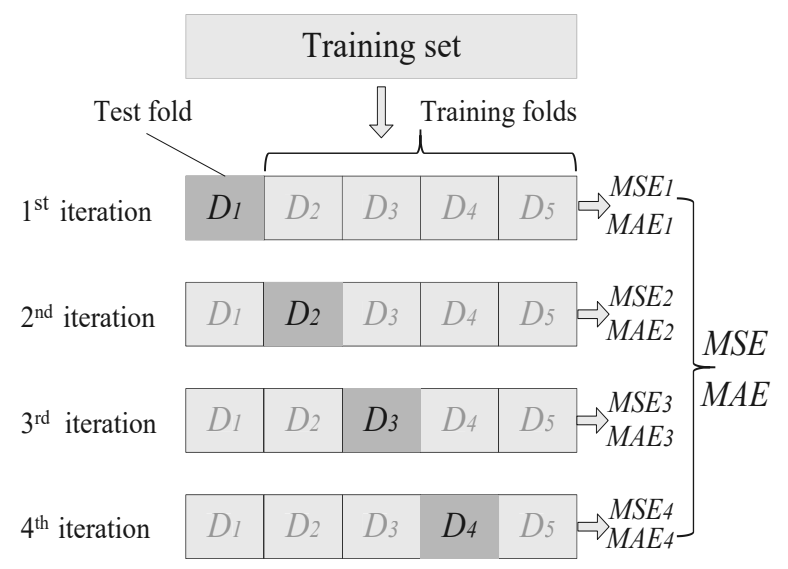

Fig. 9 Five-fold cross-validation in four iterations

The results of $K$-fold cross-validation are shown in Tab. 5, for the MSE and MAE, respectively. It can be seen from Tab. 5 that, except for the fluctuations in the MAE and MSE values of the $4^{\text {th }}$ iteration, the MAE and MSE values of the remaining three iterations have relatively small fluctuations.

Tab. 5 DNN model MF prediction values

\begin{tabular}{ccc}
\hline $\begin{array}{c}\text { Test } \\
\text { fold }\end{array}$ & MSE & MAE \\
\hline $\mathrm{D}_{1}$ & 259.7263 & 11.3574 \\
$\mathrm{D}_{2}$ & 176.0991 & 11.3406 \\
$\mathrm{D}_{3}$ & 233.6315 & 11.1843 \\
$\mathrm{D}_{4}$ & 931 & 22.8447 \\
\hline
\end{tabular}

\section{Comparison and discussion}

The MSE and MAE of MF empirical formula are shown in Eqs. (7) and (8), the MSE and MAE of the DNN MF prediction model are shown in Eqs. (9) and (10), and the $r$ of both MF empirical formula and the DNN MF prediction model are shown in Eq. (11) in combination with the calculations in Tab. 4. 


$$
\begin{gathered}
M S E=\frac{1}{9} \sum_{i=1}^{9}\left[\left(F_{X_{i}}-\hat{F}_{X_{i}}\right)^{2}+\left(F_{Y_{i}}-\hat{F}_{Y_{i}}\right)^{2}+\left(F_{Z_{i}}-\hat{F}_{Z_{i}}\right)^{2}\right] \\
M A E=\frac{1}{9} \sum_{i=1}^{9}\left[\left|\left(F_{X_{i}}-\hat{F}_{X_{i}}\right)\right|+\left|\left(F_{Y_{i}}-\hat{F}_{Y_{i}}\right)\right|+\left|\left(F_{Z_{i}}-\hat{F}_{Z_{i}}\right)\right|\right] \\
M S E=\frac{1}{4} \sum_{i=1}^{4} M S E_{i}=\frac{259.73+176.10+233.63+931}{4}=400.115 \\
M A E=\frac{1}{4} \sum_{i=1}^{4} M A E_{i}=\frac{11.36+11.34+11.18+22.84}{4}=14.18 \\
r=1-\frac{\sum_{i=1}^{9}\left[\left(\hat{F}_{X_{i}}-F_{X_{i}}\right)^{2}+\left(\hat{F}_{Y_{i}}-F_{Y_{i}}\right)^{2}+\left(\hat{F}_{Z_{i}}-F_{Z_{i}}\right)^{2}\right]}{\sum_{i=1}^{9}\left[\left(\bar{F}_{X}-F_{X_{i}}\right)^{2}+\left(\bar{F}_{Y}-F_{Y_{i}}\right)^{2}+\left(\bar{F}_{Z}-F_{Z_{i}}\right)^{2}\right]}
\end{gathered}
$$

Where:

$F_{X_{i}}, F_{Y_{i}}$ and $F_{Z_{i}}$ are milling forces in the three directions of $\mathrm{X}$ axis, $\mathrm{Y}$ axis and $\mathrm{Z}$ axis in Tab. 2, respectively $[\mathrm{N}]$,

$\widehat{F}_{X_{i}}, \widehat{F}_{Y_{i}}$ and $\widehat{F}_{Z_{i}}$ are the milling forces fitted by the multiple linear regression analysis in the directions of $\mathrm{X}, \mathrm{Y}$ and $\mathrm{Z}$ in Tab. $2[\mathrm{~N}]$, where the value of $i$ ranges from 1 to $9[-]$,

$\bar{F}_{X}, \bar{F}_{Y}$ and $\bar{F}_{Z}$ are the average values of milling forces in the directions of $\mathrm{X}, \mathrm{Y}$, and $\mathrm{Z}$ in Tab. $2[\mathrm{~N}]$,

$\mathrm{r}$ is the correlation coefficient [-].

Tab. 6 shows the calculation results of MSE, MAE and $r$ values of the MF empirical formula and the DNN MF prediction model. Three performance metrics are analyzed by comparing DNN and regression analysis. It is obvious that DNN far outperformed regression analysis for all the responses.

Tab. 6 Performance metrics comparison of regression analysis and DNN model

\begin{tabular}{cccc}
\hline & MSE & MAE & r \\
\hline regression analysis & 980.616 & 138.731 & 0.775 \\
DNN model & 400.115 & 14.18 & 0.908 \\
\hline
\end{tabular}

\section{Conclusion}

Experimental research on MF was carried out by means of a four factors and three levels orthogonal test method. According to the results of MF test in this study to predict MF of NCSBMC with regression analysis and DNN model. The findings are summarized as follows:

1) Workpiece diameter, workpiece material, milling speed and feed per tooth all have an impact on MF, with feed per tooth having the greatest impact, followed by workpiece diameter, workpiece material and milling speed.

2) According to experimental research, NCSBMC $F_{Z}$ range is $300-500 \mathrm{~N}, F_{Y}$ range is $150-200 \mathrm{~N}$, and $F_{X}$ range is $20-50 \mathrm{~N}$. In actual production, for 20 steel,
Q235B and other good processing performance with smaller diameter of low carbon steel workpiece, we can improve the feed per tooth to improve production efficiency. For 40Cr, stainless steel, die steel and other poor processing performance with larger diameter workpiece, in the premise of not affecting the production efficiency, processing parameters should choose smaller feed per tooth and higher speed.

3) A five-fold cross-validation with four iterations is implemented for the DNN model, in order to make full use of the data to test the effect of algorithm in case of insufficient sample size, which effectively avoids the occurrence of over-fitting and under-learning states, and the final fit results are useful for reliable evaluation of the model.

4) The DNN model far outperformed regression analysis for MF prediction for MAE, MSE and $r$ metrics in the absence of sufficient data. The self-learning and adaptive capabilities of DNN makes the prediction accuracy higher than that of empirical formulas. Compared with the empirical formula of linear regression analysis, the DNN has better scalability. The empirical formula fitted by the linear regression analysis needs to presuppose an exponential relationship between MF and the machining parameters, while DNN is more capable of modeling and processing non-linear models.

\section{Acknowledgments}

This research was financially supported by the National International Scientific and Technological Cooperation Special with Granted No.2018DFR50520 and by Fujian Provincial Key Project of Science and Technology with Granted No. $2019 H 0034$.

\section{References}

[1] CHANG W T., CHEN L C. (2016). Design and experimental evaluation of a circular saw blade 
with self-clamped cutting inserts. In: International Journal of Advanced Manufacturing Technology, Vol. 83, No. 1-4, pp. 365-379. ISSN 0268-3768.

[2] GUO J F. (2005). The application of metal cold cutting circular saw blade in steel rolling production. In: Metallurgical Equipment, Vol. 6, No. 1, pp. 43-48. ISSN 1001-1269.

[3] GUO S L., GU L Z., HAN J X., ZHONG C. (2018). Parameter comparison and performance analysis of new cold saw blade milling cutter and traditional saw blade milling cutter. In: Tool Technology, Vol. 52, No. 3, pp. 71-76. ISSN 1000-7008.

[4] YADAV R K., ABHISHEK K., MAHAPATRA S S. (2015). A simulation approach for estimating flank wear and material removal rate in turning of Inconel 718. In: Simulation Modelling Practice and Theory, Vol. 52, No. 1, pp. 1-14. ISSN 1569-190X.

[5] SHAN G., PENG N. (2012). Application of Improved L-M Optimization Algorithm BP Neural Network in Tool Wear Prediction. In: Macbine Tool and Hydraulics, Vol. 40, No. 15, pp. 22-26. ISSN 1001-3881.

[6] XU Z H., LI S., CHEN Q W. (2015). Consistency tracking control of multiple Euler-Lagrange systems using velocity observers. In: Control Theory and Applications, Vol. 32, No. 1, pp. 50-57. ISSN 1000-8152.

[7] S. CAMPOCASSO et al. (2017). Towards cutting force evaluation without cutting tests. In: CIRP Annals, Vol. 66, No. 1, pp. 77-80. ISSN 0007-8506.

[8] JÁNOS KUNDRÁK et al. (2018). Analysis of the Effect of Feed on Chip Size Ratio and Cutting Forces in Face Milling for Various Cutting Speeds. In: Manufacturing Technology, Vol. 18, No. 3, pp. 431-438. ISSN 1213-2489.

[9] GENG G S et al. (2020). Finite Element Analysis and Parameter Optimization Selection of High Speed Milling GH4169. In: Manufacturing Technology, Vol. 20, No. 3, pp. 300-306. ISSN 1213-2489.

[10] LI Y., XU X P. (2005). Finite Element Analysis of Saw Blade Force in Large Cutting Depth Cutting Stone. In: Chinese Journal of Engineering Macbinery, Vol. 6, No. 3, pp. 267-271. ISSN 1672-5581.

[11] HU C G., ZHANG D H., RENG J X., YANG L. (2006). Summary of Cutting Force Modeling
Methods. In: Progress in Mechanics, Vol. 4, No. 4, pp. 564-570. ISSN 1000-0992.

[12] TANG J Y., LIN X J., TANG J J., LOU J L. (2012). Force-energy simulation study of metal sawing process based on DEFORM-3D. In: Manufacturing Technology and Macbine Tools, Vol. 12, No. 8, pp. 99-103. ISSN 1005-2402.

[13] ZHU K P., ZHANG Y. (2017). Modeling of the instantaneous milling force per tooth with tool run-out effect in high speed ball-end milling. In: International Journal of Machine Tools and Manufacture, Vol. 118-119, pp. 37-48. ISSN 0890-6955.

[14] S. TURCHETTA., L. SORRENTINO. (2019). Forces and wear in high-speed machining of granite by circular sawing. In: Diamond and Related Materials, Vol. 100, ISSN 0925-9635.

[15] KAIS I. ABDUL-LATEEF AL-ABDULLAH et al. (2018). Force and temperature modelling of bone milling using artificial neural networks. In: Measurement, Vol. 116, pp. 25-37. ISSN 0263-2241.

[16] LI X., SHI Z Y et al. (2018). Artificial neural network predicts tool wear and cutting force. In: Control Theory and Applications, Vol. 35, No. 12, pp. 1731-1737. ISSN 1000-8152.

[17] WANG J C et al. (2020). Milling force prediction model based on transfer learning and neural network. In: Journal of Intelligent Manufacturing, pp. 1-10. ISSN 0956-5515.

[18] ALI YEGANEFAR et al. (2019). The use of support vector machine, neural network, and regression analysis to predict and optimize surface roughness and cutting forces in milling. In: International Journal of Advanced Manufacturing Technology, Vol. 105, No. 2, pp. 951-965. ISSN 1672-5581.

[19] HAN X., LIU Q. (2010). Modeling and analysis of PH13-8Mo plunge milling force. In: Aviation Manufacturing Technology, Vol. 24, No. 5, pp. 7280. ISSN 1671-833X.

[20] ZHU L., LU D N. (2018). Design of CNC constant force milling tool path. In: Machine Tool and Hydraulics, Vol. 46, No. 8, pp. 9-12. ISSN 1001-3881.

[21] SUN J J, KONG X etc. (2011). Experimental research on the milling force model of 6061 aluminum alloy ball end milling cutter. In: Tool Technology, Vol. 45, No. 1, pp. 22-25. ISSN 1000-7008. 